\title{
OBJECT BOUNDARIES REGULARIZATION USING THE DYNAMIC POLYLINE COMPRESSION ALGORITHM
}

\author{
Omer Saud Azeez ${ }^{1}$, Bahareh Kalantar ${ }^{2}$ *, Husam Abdulrasool H. Al-Najjar ${ }^{1}$, Alfian Abdul Halin ${ }^{3}$, Naonori Ueda ${ }^{2}$, Shattri Mansor $^{1}$
}

\author{
${ }^{1}$ Dept. of Civil Engineering, Faculty of Engineering, Universiti Putra Malaysia, Serdang, Selangor, Malaysia \\ ${ }^{2}$ RIKEN Center for Advanced Intelligence Project, Goal-Oriented Technology Research Group, Disaster Resilience Science Team, \\ Tokyo 103-0027, Japan- bahareh.kalantar@ riken.jp \\ ${ }^{3}$ Dept. of Multimedia, Faculty of Computer Science and Information Technology, Universiti Putra Malaysia, Serdang, Selangor, \\ Malaysia
}

\section{Commission VI, WG IV/1}

KEY WORDS: Building, Dynamic Polyline Compression, Segmentation, LiDAR, 3D Reconstruction.

\begin{abstract}
:
This study presents a regularization approach to refine object boundaries for the purpose of buildings 3D modelling and reconstruction. Specifically, the derivative Normalized Digital Surface model (nDSM) image layer is firstly segmented using the classical multi-resolution segmentation followed by spectral difference segmentation. As the segmentation results can contain quite a number of boundary artefacts in the form geometrical distortions, the Dynamic Polyline Compression algorithm (DCPA) is applied as a regularization step in order to refine the outer boundaries, which removes the distortions. This results in higher quality image objects for the purpose of 3D models reconstruction. Experimental results after comparing between automatically extracted buildings and manually digitized aerial photographs indicate high completeness scores of 94\%-97\% and correctness of 93\%-96\%. Overall average error is minimized with very low Root Mean Square (RMS) and Overlay errors.
\end{abstract}

\section{INTRODUCTION}

Light detection and Ranging (LiDAR) has been widely used in the remote sensing community. This is mainly because such data can be obtained over large areas in a relatively short period of time (Kwan et al., 2010). LiDAR instruments normally include a global positioning system (GPS) receiver for positioning, an Inertial Measurement Unit (IMU) for angular measurements, and a laser for distance- and position-based calculations of a point from land surfaces (Höfle and Rutzinger, 2011). The advantages of using LiDAR data are its high speed, high density, high vertical accuracy and low cost compared to traditional photogrammetry. LiDAR systems are becoming more widespread in remote sensing with applications in 3D reconstruction, specifically the feature extraction phase (Wei., 2008).

Buildings take up a majority of the terrain in a city. In remote sensing, building boundary determination is a crucial and challenging task, especially for 3D building reconstruction (Rottensteiner and Briese, 2002). Several attempts have been made to reliably detect building boundaries. For example, Ma (2004) applied image segmentation on the Normalized Digital Surface Model (nDSM) raster layer to extract boundaries by fine tuning an elevation threshold in order to exclude useless features. In the study by Sampath and Shan (2004), they performed regularization post segmentation for the reconstruction of buildings shapes using a region growing algorithm. Xinlian (2005) applied the Smallest Deviation Approximation algorithm to LIDAR to extract building boundaries. They initially discovered the two farthest points

* Corresponding author within the building area in order to divide the building into two smaller parts. This process is iterated until the similarity distance is lesser than a specified threshold.

Most previous efforts have focused on building extraction and boundary determination. However, there is also the issue where extracted building boundaries are not smooth and contain distortions. This paper focuses on these issues, for the purpose of buildings 3D reconstruction. Specifically, we perform building boundary extraction that is assisted by a regularization step that removes distortions/artefacts from building boundaries. Following data pre-processing, our approach consists of three stages. First, generated nDSM images are segmented using the multi-resolution segmentation followed by spectral difference segmentation. Second, regularization is performed using the Dynamic Polyline Compression algorithm (DPCA) on the segmentation results for outer boundaries refinement, which is followed by an object-based image analysis (OBIA) classification step. Finally, regions classified as buildings are modelled in the $3 \mathrm{D}$ space based on the extracted building boundaries.

\section{STUDY AREA AND DATA USED}

This study was conducted over the urban area of Serdang, Selangor, Malaysia. Geographically, the study area is located within the Universiti Putra Malaysia campus, between $101^{\circ} 42^{\prime}$, $101^{\circ} 43^{\prime} \mathrm{E}$ and $3^{\circ} 0^{\prime} 9^{\prime \prime}, 3^{\circ} 0^{\prime} 27^{\prime \prime} \mathrm{N}$. This study area is characterized by buildings with different heights with the presence of tall trees and vegetation, and small lakes. There is also the road network that connects the university with the surrounding areas. The surface area of the test sample is approximately $1 \mathrm{~km}^{2}$, and is illustrated in Fig. 1 . 
The LiDAR point clouds data was collected by an airborne LiDAR system on March 8, 2015. The camera has a spatial resolution of $10 \mathrm{~cm}$ and the laser scanner has a scanning angle of $60^{\circ}$ with a camera angle of $\pm 30^{\circ}$. The posting density of the LiDAR data was 3-4 pts $/ \mathrm{m}^{2}$ (average point spacing $=0.41 \mathrm{~m}$ ). The minimum and maximum elevations are 36 and $69 \mathrm{~m}$, respectively.

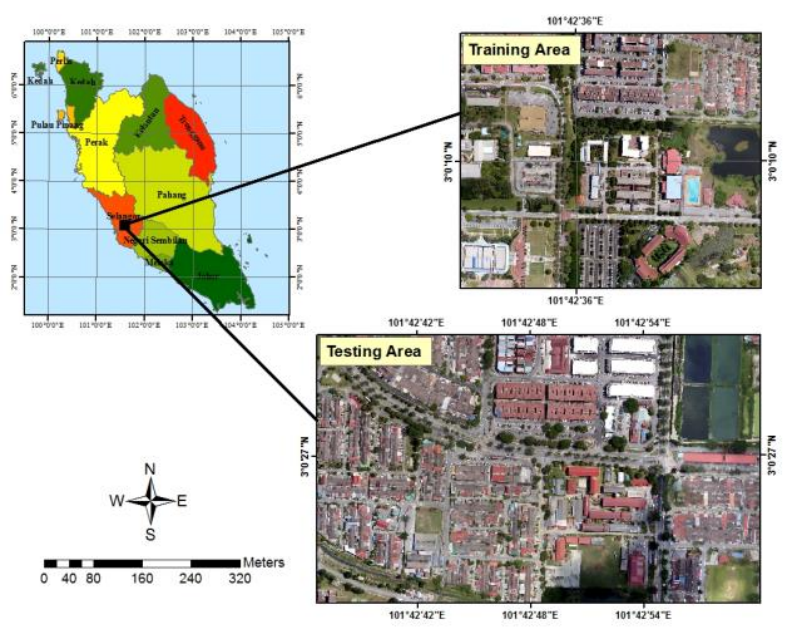

Figure 1. The study area.

\section{METHODOLOGY}

The proposed methodology consists of three (3) main stages. Note that these stages are executed after data pre-processing (Section 3.1). The main stages are:

1. Segmentation: Each nDSM layer is firstly segmented into spectrally similar regions to allow OBIA. This is crucial so that the image is partitioned into regions that are deemed semantically significant. Such regions commonly share a homogenous trait such as being spectrally similar. Two segmentation algorithms are applied namely multi-resolution segmentation followed by spectral difference segmentation.

2. Regularization and OBIA-based classification: Weih and Riggan (2010) showed that OBIA outperforms pixel-based methods for multi-spectral images. OBIA is also faster since whole regions are considered instead of individual pixels (Kalantar et al., 2017b; Ahmed et al., 2017). Similarly, our work adopts an OBIA-based classification approach where the regions are classified into building, tree or noise. Note that many of the segmented regions contain artefacts (i.e. jagged edges/artefacts). To alleviate this problem, we apply the DPCA before performing OBIA classification in order to refine each region. This assumedly will produce better extracted regions.

3. 3D modelling and reconstruction: Ideally, the extracted building boundaries should be smooth polygons. The output from step (2) is then used as a reference to reconstruct and model the buildings in 3D space.

The proposed technique was developed using the eCognition (Trimble Geospatial) and ArcGIS10.3 applications where the overall flow is shown in Fig 2.

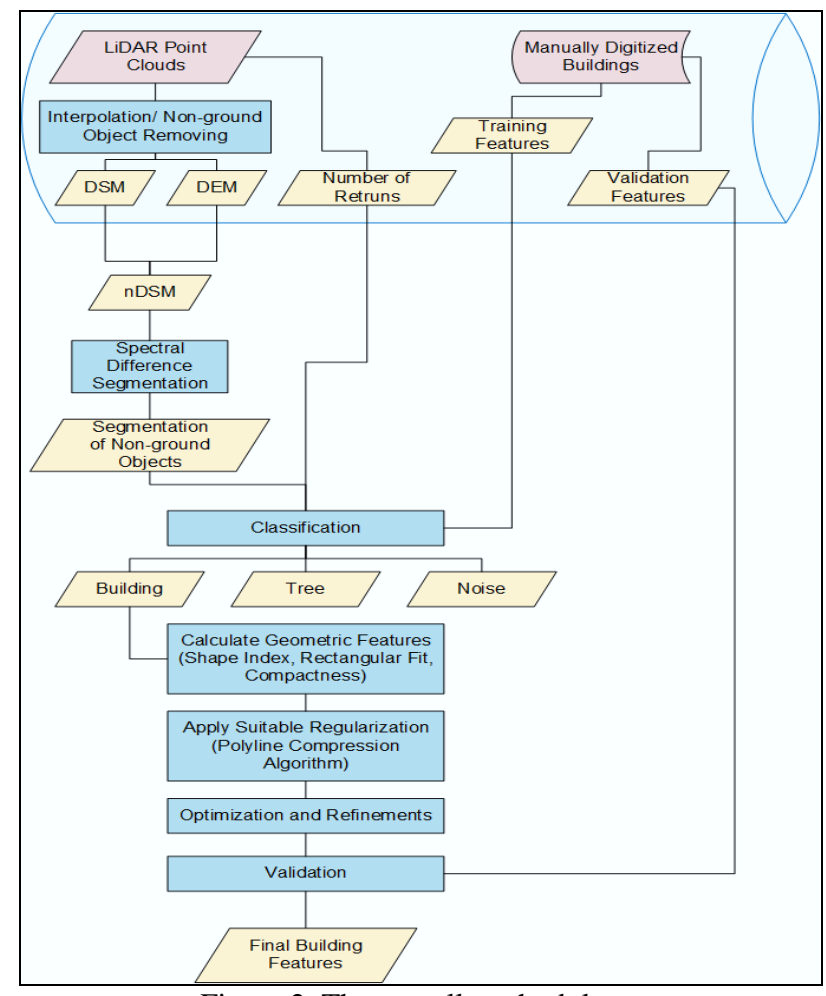

Figure 2. The overall methodology.

\subsection{Data pre-processing}

Several pre-processing steps are necessary in order to prepare the data for the main processing and analysis. Firstly, the LiDAR point cloud data is converted into the LAS (LASer) extension. Data with regards to laser returns are recorded from ground and non-ground target strokes. To derive the required digital elevation model (DEM), only returns from bare-earth are considered where the data needs to be separated through the filtration process in Zhao et al. (2007). Using the Inverse Distance Weighing (IDW) interpolator, terrain points are processed to generate the DEM layer and surface points are processed to generate the Digital Surface Model (DSM) layer. Sample results are shown in Figs. $3 a$ and $3 b$. Since the nDSM is basically the absolute elevation of artificial elevation features with regards to the earth, it is derived by subtracting the DEM layer from the DSM layer using the map algebra tool in ArcGIS 10.3. This is represented in Eq. 1.

$$
n D S M=D S M-D E M
$$

Notice the different colored pixels in the nDSM (Fig. 3c). This depicts the different objects' heights from the terrain, which renders the nDSM useful for buildings classification as height and other parameters are available. 

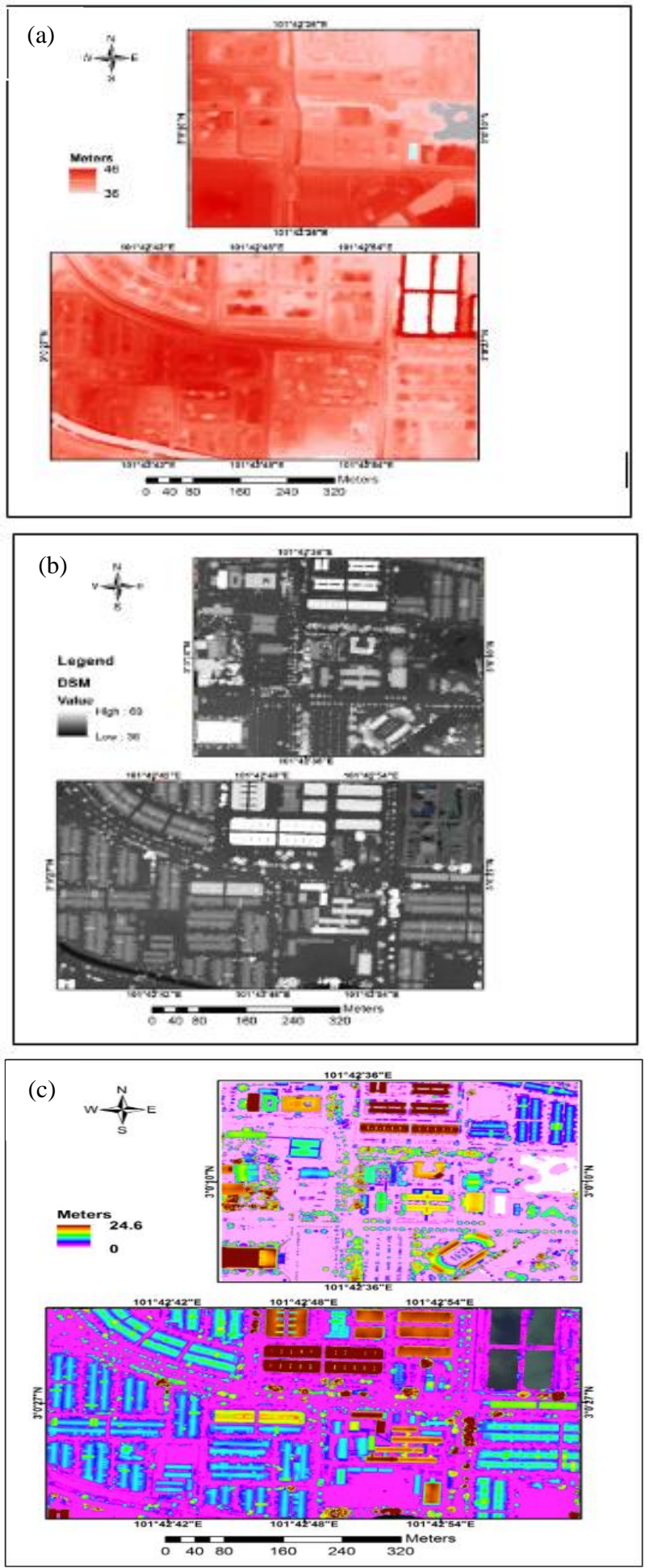

Figure 3. Laser scanning data derivatives (a) DEM, (b) DSM,

(c) nDSM.

\subsection{Segmentation}

As mentioned, OBIA works on segmented regions within an image. Therefore, we firstly perform multi-resolution segmentation where the algorithm iteratively groups image objects until the upper object variance limit is reached. Fractal borders of each segmented region is minimized by weighting the scale parameter with the separation of shape and compactness parameters. Next, spectral difference segmentation (SDS) is applied to further refine the segmentation results. SDS merges neighboring image objects whose spectral means fall below a specified threshold. In our work, the threshold that yielded best results (representing maximal spectral difference) is 12 . Applying two segmentation steps is motivated by the work of Ostdijk et al., (2008) where decent segmentation results were consistently obtained across their dataset. Figure 4 illustrates sample results after segmentation using multi-resolution and spectral difference segmentations.

\subsection{Regularization and OBIA-based Classification}

In order to refine any undesirable artefacts on the polygons generated from the segmentation process, we performed a regularization step by applying the dynamic polyline compression algorithm (DPCA). Besides refining region edges, DPCA also reduces the number of over-segmented polygons (while avoiding under-segmentation), which decreases processing time. The algorithm works by removing vertices causing the resultant polyline to pass through a subset of the source vertices (Gribov 2015). Figure 5 shows the effects of applying the DPCA on the polygons.

Once regularized, classification can be performed. Each of the segmented regions is classified using the Random Forest (RF) classification algorithm, into one of the following classes: building, tree or noise. RF is an ensemble learning technique that generates a multitude of random decision trees that are then aggregated to compute a classification (Breiman, 2001). The RF classifier was chosen since it involves few user-defined parameters to yield the best overall accuracy levels. These accuracy levels are either comparable to or better than other classifiers such as Maximum Likelihood and conventional Decision Trees (Lawrence et al., 2006), AdaBoost decision trees and neural networks (Chan and Paelinckx., 2008), and Support Vector Machines (Pal., 2005; Kalantar et al., 2017a). 25 samples for each class were selected to train the RF algorithm. The classification algorithm was applied to the whole study area and all image objects were then classified into: building and trees or noise. Basic information of study area is needed in order to have an efficient classification. In this study, an aerial photo is used to identify the ground features (i.e. Land use/cover). Geometry features namely, shape index rectangular fit, compactness was used for building extraction (Kibret 2016). Buildings Detected based on the calculation of feature's geometry (shape index, rectangular fit and compactness) in order to prepare this layer to the final step of the extraction process (Kibret 2016). The classification results are evaluated using the confusion matrix (Table 1). This matrix was then used to calculate the overall accuracy and kappa index. The overall accuracy and kappa index were estimated as $91.9 \%$ and 0.891 , respectively.

\subsection{D Modelling and Reconstruction}

After the regularization step on the building boundaries, the " $Z$ " dimension of LiDAR data can be used to obtain the elevations of each segmented region. The 3D visualization of buildings with simple flat, multi-level flat or complex roofs achieved by segmenting portions of roofs whose " $Z$ " share similar values. A sample of reconstructed 3D buildings is shown in Figure 8. 

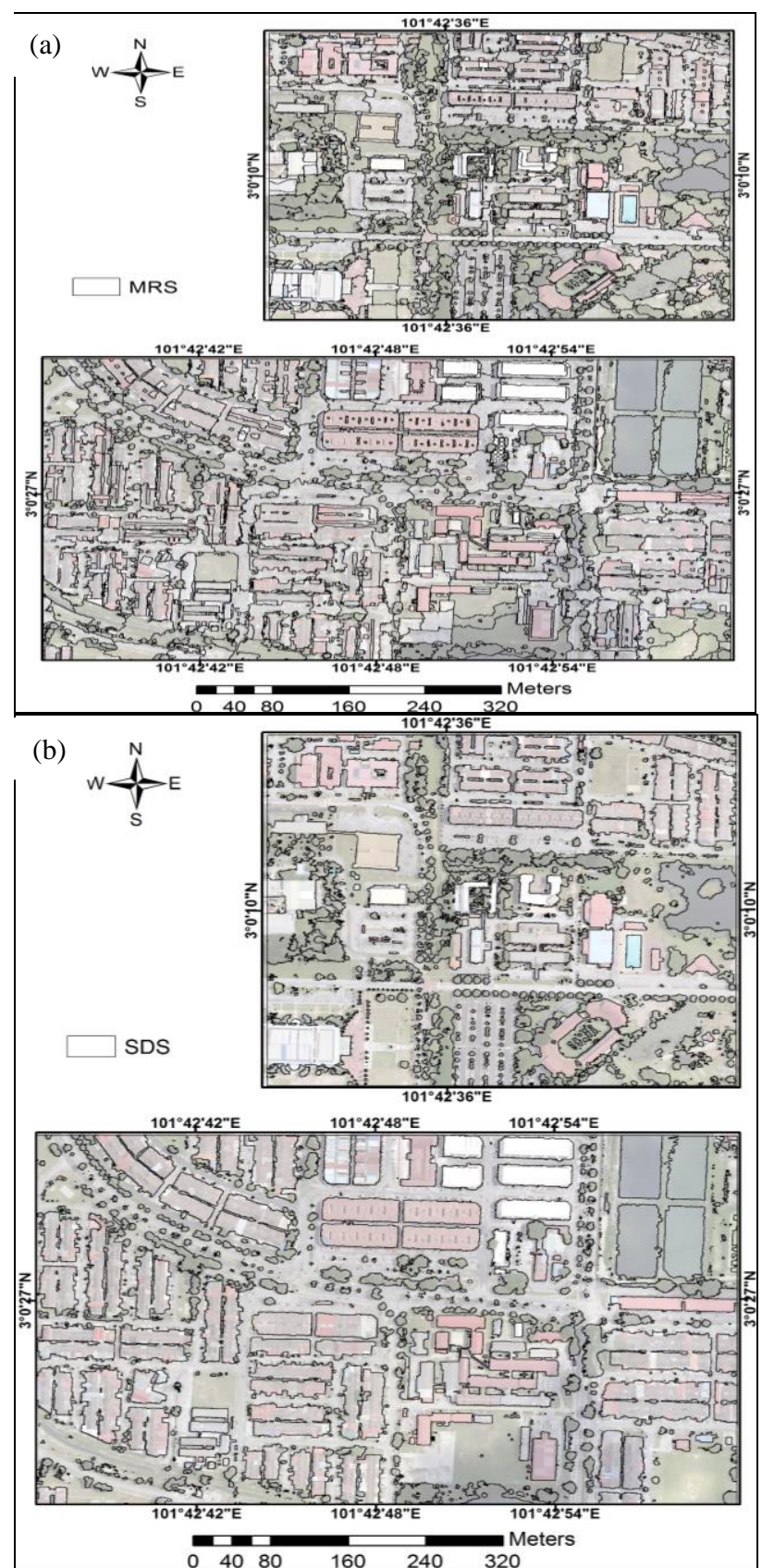

Figure 4. The results after applying a) multi-resolution segmentation, b) spectral difference segmentation.

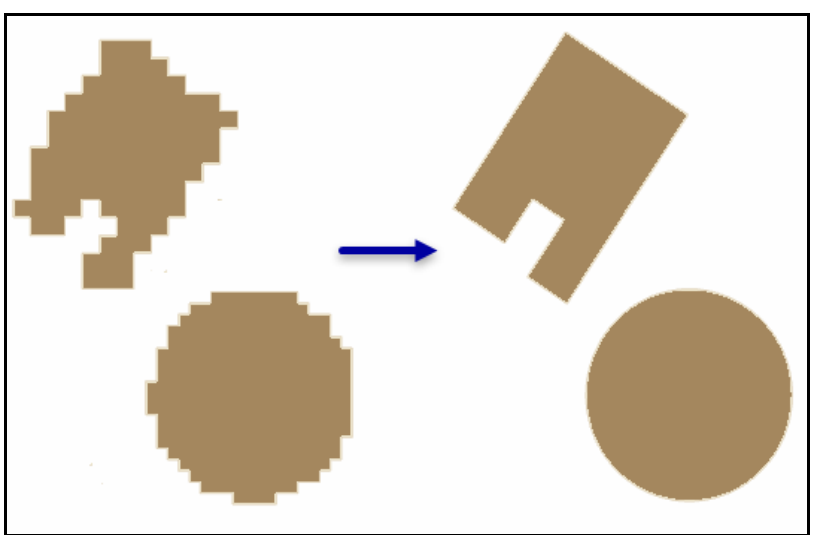

Figure 5. Effects of applying the dynamic polyline compression algorithm (Adapted from: https://goo.gl/9jrrRw)

\begin{tabular}{|c|c|c|c|}
\hline \multicolumn{4}{|c|}{ Ground Truth (Pixels) } \\
\hline Class & Buildings & Trees & Total \\
Unclassified & 262 & 47 & 309 \\
Buildings & 6501 & 0 & 6501 \\
Trees or noise & 2 & 5103 & 5105 \\
Total & 6765 & 5150 & 11915 \\
\hline \multicolumn{4}{|c|}{ Ground Truth (Percent) } \\
\hline Class & Buildings & Trees & Total \\
Unclassified & 3.87 & 0.92 & 24.56 \\
Buildings & 96.1 & 0 & 41.93 \\
Trees & 0.03 & 99.67 & 35.07 \\
Total & 100 & 100 & 100 \\
\hline \multicolumn{2}{|c|}{0.91} \\
\hline \multicolumn{2}{|c|}{ Overall Accuracy Coefficient }
\end{tabular}

Table 1. The confusion matrix and accuracy scores for the classification step.

\section{RESULTS}

We initially present the results of regularization after DCPA. The regularization allowed extraction of the most compact and refined regions after both multi-resolution and spectral difference segmentations. This is mainly because DCPA reduced the number of over segmented regions, while avoiding under segmentation. Figure 6 shows sample results of applying DCPA on our dataset. It can be clearly seen that oversegmented regions have been eliminated. It is also observed that the outer boundaries of the buildings are still well preserved, which will in turn greatly facilitate the classification stage (and hence, extraction). Ultimately, it can also be expected that the 3D models being reconstructed will be better. Figure 7 shows the final extraction results from LiDAR data and Figure 8 showing the reconstructed $3 \mathrm{D}$ building models. The $3 \mathrm{D}$ modelling was made possible by using the average height of the area occupied by the respective boundaries. It can be seen that the building is classified based on their roof type into three classes namely, complex, multi-level flat, and simple flat. The block model is sufficient for our application because it provides a good basis for visualization, which in turn can be useful for spatial planning and disaster scenario modelling. 
(a)

(b)

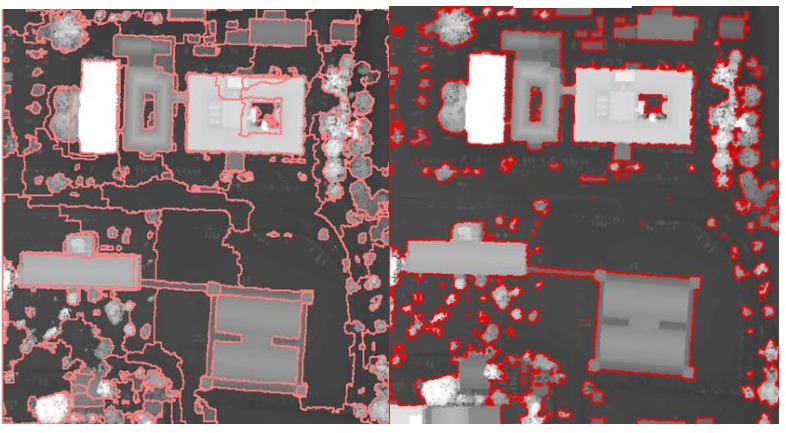

(c)

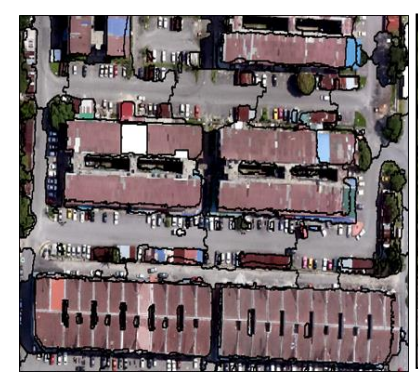

(d)

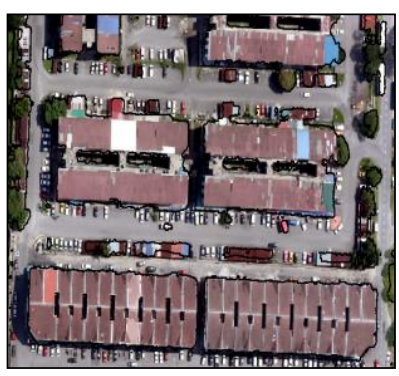

Figure 6 . The segmentation results (a), (c) before regularization and (b), (d) after regularization.

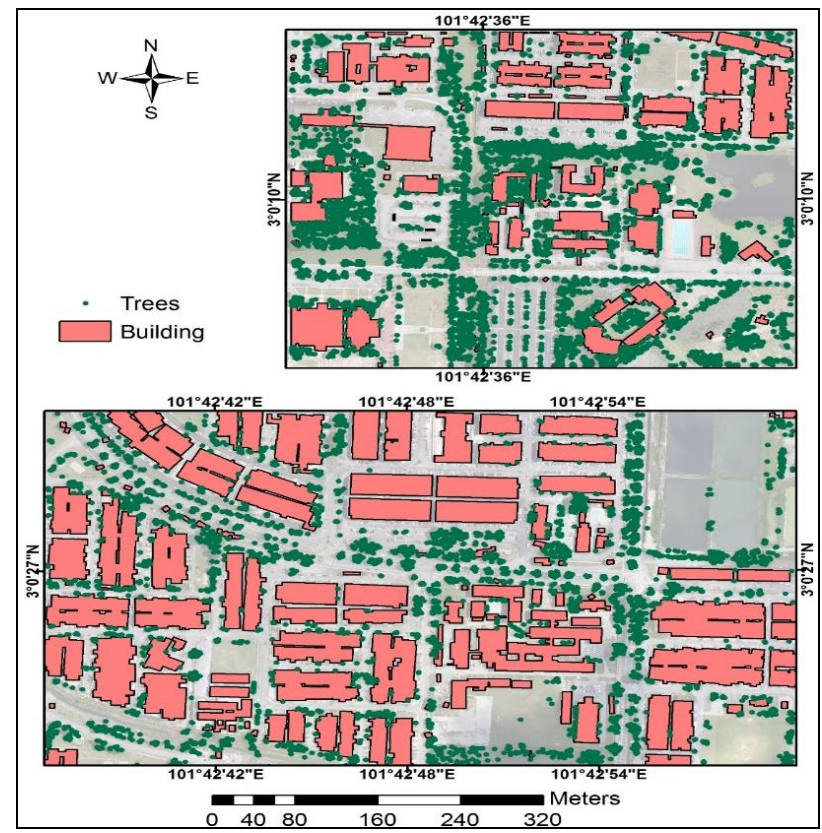

Figure 7. The classification result from LiDAR data.

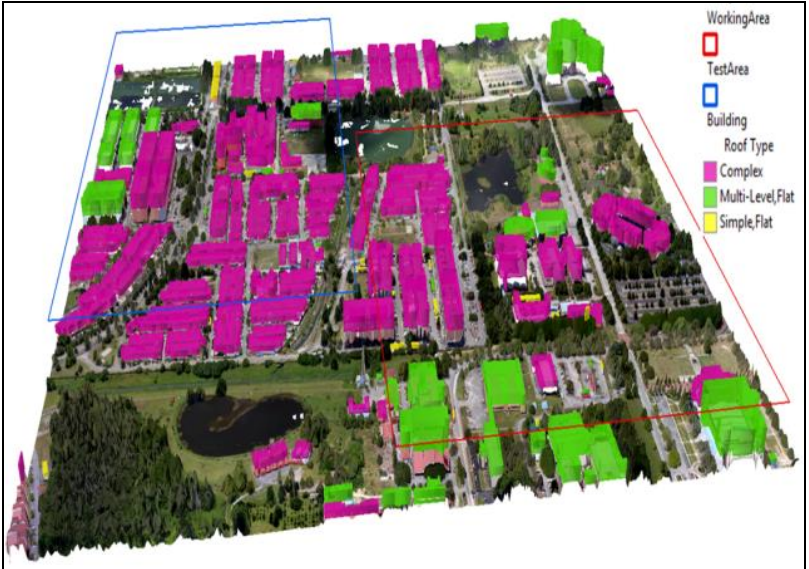

Figure 8. 3D model with classification based on building roof type.

\subsection{Accuracy assessment}

Accuracy assessment was applied by comparing between the extracted boundaries and the reference data of the manually digitized aerial photos of the buildings' boundaries (at $10 \mathrm{~cm}$ resolution). The four quality measures of Completeness, Correctness, Root Mean Square (RMS) error and overlay error are used to assess the accuracy for the training and validation datasets. Completeness represents the percentage of correct extraction whereas RMS error is related to geometrical accuracy. Area difference and overlay errors are used to determine the shape similarity between the buildings (Haithcoat et. al, 2001).

Completeness and correctness are calculated by comparing between the number of extracted buildings and number of buildings in the reference data. Horizontal RMS error is calculated as the distances between the corresponding building corners. Overlaying the extracted buildings on top of the reference data leads to the discovery overlay errors as well as area and perimeter difference measurements. Since the reference data does not have height information, vertical geometric accuracy is not assessed.

The calculated quality measures are listed in Table 2 .

\begin{tabular}{|c|c|c|}
\hline & Area 1 & Area2 \\
\hline Total building number & 89 & 45 \\
Completeness & $94 \%$ & $97 \%$ \\
Correctness & $93 \%$ & $96 \%$ \\
RMS Error & $0.8 \mathrm{~m}$ & $0.7 \mathrm{~m}$ \\
Overlay error & $13 \%$ & $4 \%$ \\
\hline
\end{tabular}

Table 2. The accuracy assessments for building extraction.

\section{DISCUSSION}

In this study, the accuracy of building extraction is performed through segmentation algorithms (multiresolution and spectral difference) and DCPA based on LiDAR point cloud data. The proposed methodology is capable of producing a $2 \mathrm{D}$ building polygon and $3 \mathrm{D}$ building models. $3 \mathrm{D}$ building models or $2 \mathrm{D}$ building polygons are used in most of web-based maps and 3D navigation maps. In this context, the proposed approach will be useful to update building information of maps. The comparison 
between buildings boundaries as shown in Figure 6 shows promise that the regularization process of DCPA adds value for the final extraction. Additionally, corner points of buildings seem to appear at their proper locations, even if the original dataset did not contain points at these positions.

\section{CONCLUSION}

The main objective of this research is to study the effects of regularization in the buildings region extraction workflow. The regularization algorithm used in this work is DCPA, which is applied after segmentation. Results show that building boundaries containing geometrical distortions are refined using DCPA. Image objects are also properly segmented where over and under-segmentation are avoided. Classification results are promising where accuracy is 0.91 and RMS and overlay errors are kept low. Furthermore, compactness and correctness scores are also promising at $93 \%-97 \%$. It is worth noting that the dataset used in this work consists of complexly shaped buildings. The proposed approach however still manages to produce robust boundaries. 3D models generated from the extracted buildings are also presented. In future work, we plan to extend the approach to extract 3D building models from terrestrial LIDAR data and components with more complex shapes will be detected and regularized.

\section{REFERENCES}

Ahmed, A.A., Kalantar, B., Pradhan, B., Mansor, S. and Sameen, M.I., 2017, July. Land Use and Land Cover Mapping Using Rule-Based Classification in Karbala City, Iraq. In Global Civil Engineering Conference (pp. 1019-1027). Springer, Singapore.

Breiman, L., 2001. Random forests. Machine learning, 45(1), pp.5-32.

Chan, J. C. W., \& Paelinckx, D. (2008). Evaluation of Random Forest and Adaboost tree-based ensemble classification and spectral band selection for ecotope mapping using airborne hyperspectral imagery. Remote Sensing of Environment, 112(6), 2999-3011.

Gribov, A., 2017, November. Searching for a compressed polyline with a minimum number of vertices. In Document Analysis and Recognition (ICDAR), 2017 14th IAPR International Conference on (Vol. 2, pp. 13-14). IEEE.

Haithcoat, T.L., Song, W. and Hipple, J.D., 2001. Building footprint extraction and 3-D reconstruction from LIDAR data. In Remote Sensing and Data Fusion over Urban Areas, IEEE/ISPRS Joint Workshop 2001 (pp. 74-78). IEEE.

Höfle, B. and Rutzinger, M., 2011. Topographic airborne LiDAR in geomorphology: A technological perspective. Zeitschrift für Geomorphologie, Supplementary Issues, 55(2), pp.1-29.

Kalantar, B., Mansor, S.B., Sameen, M.I., Pradhan, B. and Shafri, H.Z., 2017a. Drone-based land-cover mapping using a fuzzy unordered rule induction algorithm integrated into objectbased image analysis. International journal of remote sensing, 38(8-10), pp.2535-2556.
Kalantar, B., Mansor, S.B., Shafri, H.Z.M. and Halin, A.A., $2017 \mathrm{~b}$. Integration of template matching and object-based image analysis for semi-automatic oil palm tree counting in UAV images. In Asian Conference on Remote Sensing (ACRS), Sri Lanka.

Kibret, K.S., Marohn, C. and Cadisch, G., 2016. Assessment of land use and land cover change in South Central Ethiopia during four decades based on integrated analysis of multitemporal images and geospatial vector data. Remote Sensing Applications: Society and Environment, 3, pp.1-19.

Kwan, M.P. and Ransberger, D.M., 2010. LiDAR assisted emergency response: Detection of transport network obstructions caused by major disasters. Computers, Environment and Urban Systems, 34(3), pp.179-188.

Lawrence, R.L., Wood, S.D. and Sheley, R.L., 2006. Mapping invasive plants using hyperspectral imagery and Breiman Cutler classifications (RandomForest). Remote Sensing of Environment, 100(3), pp.356-362.

Ma, R., 2005. Building model reconstruction from LiDAR data and aerial photographs (Doctoral dissertation, The Ohio State University)

Oostdijk, A., Persie, M.V., Noorbergen, H.H.S. and Van Rijn, J.W., 2008. Multi scale object based detection and classification of roads and vehicles in high resolution optical satellite imagery.

Pal, M., 2005. Random forest classifier for remote sensing classification. International Journal of Remote Sensing, 26(1), pp.217-222.

Rottensteiner, F., Briese, C., 2002, A New Method for Building Extraction in Urban Areas From High-Resolution Lidar Data, International Archives of Photogrammetry Remote Sensing and Spatial Information Sciences, 34(3/A), pp.295-301.

Sampath, A. and Shan, J., 2004. Urban modeling based on segmentation and regularization of airborne LIDAR point clouds. In XXth ISPRS Congress.

Wei, S., 2008. Building boundary extraction based on lidar point clouds data. Proceedings of the International Archives of the Photogrammetry, Remote Sensing and Spatial Information Sciences, 37, pp.157-161.

Weih, R.C. and Riggan, N.D., 2010. Object-based classification vs. pixel-based classification: Comparative importance of multiresolution imagery. The International Archives of the Photogrammetry, Remote Sensing and Spatial Information Sciences, 38(4), p.C7.

Xinlian, L., 2005. Airborne LIDAR Data Filtering and Building Model Reconstruction. Dissertation, Chinese Academy of Surveying and Maping, Beijing, China.

Zhao, L., Lai, Z., Li, Y., Xue, Y., Liao, M., Wu, Z., Liu, P. and Liu, X., 2007. Application and analyses of airborne LiDAR technology in topographic survey of tidal flat and coastal zone. In Proceedings of the 1st North America Landslide Conference. Association of Engineering Geologists Special Publication (pp. 699-702) 